\title{
END-TO-END INFORMATION SYSTEM DESIGN \\ AT THE NASA JET PROPULSION LABORATORY
}

\author{
Adrian J. Hooke \\ Technical Group Supervisor \\ NASA Jet Propulsion Laboratory \\ 4800 Oak Grove Drive \\ Pasadena, CA 91103, USA
}

\begin{abstract}
Recognizing a pressing need of the 1980's to optimize the two-way flow of information between a ground-based user and a remote space-based sensor, an end-to-end approach to the design of information systems has been adopted at the Jet Propulsion Laboratory. The objectives of this effort are to ensure that all flight projects adequately cope with information flow problems at an early stage of system design, and that cost-effective, multimission capabilities are developed when capital investments are made in supporting elements.
\end{abstract}

This paper reviews the End-to-End Information System (EEIS) activity at the Laboratory, and notes the ties to the NASA End-to-End Data System program.

\section{INTRODUCTION}

The Jet Propulsion Laboratory is heavily involved with the exploration of deep space (e.g., the Mariner series of spacecraft to Mars, Venus, and Mercury; the Viking series to Mars; the Voyager series to Jupiter and Saturn; and the Galileo series to Jupiter), and has recently become involved again with earth-orbiting missions such as the SEASAT-A global ocean dynamics monitoring spacecraft and the Infra-Red Astronomical Satellite (IRAS).

As the nation's involvement in space has matured, the demands of the investigator (user) community for greater data return, faster telemetry delivery, and more responsive sequencing have increased. Whereas the early exploration missions were content to blaze the trail with a limited number of TV pictures and a few nonimaging measurements, today's spacecraft are sophisticated and highly-complicated remote laboratories, equipped with powerful on-board computational resources, and are able to return hundreds of thousands of bits per second of data from our neighboring planets. In order to use this 
resource efficiently, the user requires the capability to process telemetered measurements rapidly, and to interact with his sensor - for instance, to retarget an upcoming picture sequence — via the sequencing and command processes.

The transfer function between the sensor and the user (Figure 1) is a bidirectional information system. Measurements of a remote phenomenon are telemetered to the user on the ground for his analysis and interpretation, and he responds by adjusting the parameters of upcoming sensing activities by commanding mode or by pointing changes to the instrument. When the sensor and the user are both colocated in a laboratory during prelaunch checkout, the transfer function is hardwired and straightforward. As the sensor is progressively separated from the user - during integration with a spacecraft system, launch, and transfer to a remote measuring station in space - the transfer function becomes correspondingly more complex, involving both digital processing elements and analog (radio) transmission paths. A measure of the effectiveness of the information system is the degree to which the transfer function remains transparent to the user (other than the inevitable propagation delay) as this separation increases from zero to many millions of kilometers.

In reality, the transfer function involves several resource-constrained elements spacecraft systems, bandwidth-limited RF links, ground-tracking stations, control centers, etc. - each of which contributes some local degradation to the efficiency of information flow. Since most of the elements represent large capital investments for NASA, their designs tend to evolve in directions which provide multimission capabilities of general utility to all projects. Clear challenges therefore exist to steer these multimission developments along directions which are the most useful to all projects and also to "system-engineer" the information flow for a particular project in order to satisfy the requirements of the end user in the most cost-effective manner.

Therein lies the key to the system approach: The user is the most important element in the system. It is he who judges the success of the mission, who interprets the results for public consumption, and who provides prime movement in the fiscal approval of new projects. The intermediate elements of the information system have no self-sustaining functions other than to expedite the exchange of information between the user and his remote sensor.

Recognizing this need, a formal discipline of EEIS engineering has been created at JPL. System designs, which cross both technical and organizational boundaries, are being developed with a primary view towards optimizing the end-to-end efficiency and costeffectiveness of information flow for our space missions. 


\section{SYSTEM ELEMENTS}

The transfer function identified in Figure 1 has been subdivided into seven major discipline elements for the purpose of system comprehension. These elements are either associated with direct pipeline or "along-path" flow of a single information type between the sensor and the user or are involved with "cross-path" functions, which tap into more than one pipeline. This is illustrated in Figure 2. Although tailored to the configuration of JPL space missions, the figure could probably be easily adapted to any complex information system.

During typical JPL missions, the ends of the EEIS are the remotely-sensed space phenomenon and the user of the sensed information on the ground respectively. For scientific missions, the user is normally a single Principal Investigator or a small team of investigators who are concerned with a single sensor. Applications missions (generally in earth orbit) tend to have a multifaceted user community, consisting of many disciplineoriented consumers of information, who require access to several sensors.

The information system which services the "ends" has three basic along-path elements:

- A Telemetry Delivery element, which is dedicated to the transport of data from the sensor down to a data base on the ground. This element does not normally interface with a user without some intermediate extractive processing or formalization into a data record.

- A Sequence and Command Delivery element, which is dedicated to the assembly and validation of a desired sequence of spacecraft activities and the resultant delivery of command stimuli to the remote sensor.

- A Tracking and Radio Science element, devoted to utilization of the RF telecommunications links between the earth and a remote spacecraft, to determine the spacecraft ephemeris and scientific phenomena of the transmission paths.

The system also has four cross-paths which, in addition to performing major internal functions, have significant interfaces with the along-paths:

- A Data Records element, devoted to the assembly and delivery of archival-quality data products in response to the Laboratory's commitments to users. While telemetry is the most common deliverable, tracking and radio science records are also produced. The system also accesses all of the along-path data to produce supplementary information, which defines such things as the spatial location of each measurement, sensor mode, and the configuration of each major EEIS element. 
- An Information Processing element, which operates primarily on delivered telemetry data for a variety of reasons including:

- $\quad$ Real-time data processing in support of spacecraft and sensor performance monitoring.

- $\quad$ Non-real-time processing to produce summaries as an aid to spacecraft troubleshooting or long-term subsystem evaluation.

- Near or non-real-time processing which reduces raw sensor data to a primary extracted information type (e.g., converts to engineering units, derives a physical quantity, etc.) in support of the user.

The Information Processing element may interface directly with the user or may output to Data Records when formal deliverables are involved.

While "downlink" processing may predominate, the Information Processing element also has an important "uplink"-related role by providing:

- A medium for the user to access the sequencing system in order to input requests for upcoming sensor activity.

- A medium for the user to access the information processing element itself and to request changes in processing parameters.

- A Mission Operations element, which controls all aspects of the EEIS during conduct of the mission. This element establishes basic requirements for the way in which missions will be conducted and also injects operability and testability into the EEIS design.

- A Spacecraft Checkout element, which defines the unique requirements on the EEIS imposed by the prelaunch integration and test of the space vehicle.

It should be noted that this functional zoning of the information system does not include one important ingredient, namely, the identification of specific facilities, equipment, or organizations that define physical configuration. If Figure 2 is redrawn in terms of support complexes rather than information flow disciplines, then the picture shown in Figure 3 emerges. One striking conclusion which emerges from examination of Figures 2 and 3 is that virtually all project support organizations are "cross-path" in nature. Examples of this are spacecraft designers, the tracking stations of the Deep Space Network (DSN), and the operations complex and computational resources of the Mission Control and Computing Center (MCCC). This is typical of most NASA arrangements. Each organization provides path-oriented support internally; by virtue of prudent engineering from all parties, major problems seldom occur at the interfaces. Such an arrangement is, however, extremely 
vulnerable to local data damage as information is moved through one of the "along-path" pipelines, where this damage may have unforeseen consequences downstream. An example of this might be a telecommunications link that introduces errors with a distribution that is catastrophic to a sensor whose data set is not properly protected. To eliminate such disasters at the user level, it is vital that the end-to-end transport of data be engineered from an overall systems viewpoint.

\section{SYSTEMS APPROACH}

As previously noted, there are two complementary facets to the EEIS activity. The first is the need to engineer the information flow for a particular project in the most effective and user-responsive manner possible. The approach taken at JPL has been to create project dedicated EEIS Design Teams for each new mission whose sole task is to perform a systems design to satisfy the information transfer requirements of that mission. Each team is led by "along-path" systems engineers and is supported by "cross-path" representatives of each supporting organization. The output of the team is a set of functional requirements for overall information transfer, which are then apportioned among the spacecraft systems and support organizations for implementation. This approach has already been successfully applied to the SEASAT, IRAS, and Galileo projects.

The second (and perhaps more difficult) task is to perform a multimission EEIS design which can be used to support a variety of projects. Cost-effectiveness is the primary driver for this approach: The more a project can adapt to a multimission capability that exists on the spacecraft and/or the ground, the smaller the cost that the project will incur to obtain support. Lowering the project cost could theoretically enable more projects to be started, and economies of scale could be achieved.

At the time of writing this paper, a serious attempt is being launched to perform a multimission systems design of the entire JPL EEIS for flight projects. The approach envisaged is to perform a coordinated top-down design of each key discipline element, following the general structure shown in Figure 2. Each element will define a total systems architecture having high multimission potential, which will subsequently be evaluated by the support organizations and traded off for overall implementation cost versus benefits achieved. Our goal is to influence significantly the next generation of multimission support capabilities, which, in practice, translates into all post-Voyager developments.

\section{RELATIONSHIP TO THE NEEDS PROGRAM}

The NASA End-to End Data System (NEEDS) Program is an intercenter NASA thrust to develop the technologies which enable dramatic improvements in the overall efficiency and productivity of information transfer within the Agency. Clearly the NEEDS activities and 
the JPL-EEIS efforts are closely aligned.

In one particular area - the delivery of telemetry from a remote sensor to a user-oriented data base on the ground - clear multimission potential exists. Both JPL and the Goddard Space Flight Center (the lead center for NEEDS) are actively pursuing the standardization of telemetry formats into a common packet protocol (1)(2)(3). This activity is summarized by another paper (4) presented at this session of the Instrument Society of America Conference. Under the NEEDS technology umbrella, we are also sponsoring the development of new telemetryhandling techniques (such as advanced channel-coding applications), which we hope will soon manifest themselves in upcoming JPL missions.

\section{CONCLUSION}

For NASA, the 1980's promise to be a decade dominated by a data rate explosion and by our ability to cost-effectively transfer information to science or applications users. The users are our customers, and we can only satisfy their requirements by prudent and thorough design of the information systems which we provide to serve them. At the Jet Propulsion Laboratory we are tackling the problems of end-to-end system design not only at the individual project level, but also from a multimission viewpoint. As a formal discipline, EEIS engineering is young (less than two years), but already significant strides have been made in understanding and solving the problems of information flow between the sensor and the user.

\section{ACKNOWLEDGMENTS}

The research described in this paper was carried out at the Jet Propulsion Laboratory, California Institute of Technology, under NASA Contract NAS7-100.

\section{REFERENCES}

1. Ferris, Albert G. and Greene, Edward P., "A Proposed Concept for Improved NASA Mission Data Management," Report X-533-76-81, Goddard Flight Center, Greenbelt, Maryland, USA, October 1976.

2. Greene, Edward P., "A Proposed Asynchronously Multiplexed Telemetry System for Spaceflight Operations," Report X-533-77-221, Goddard Space Flight Center, Greenbelt, Maryland, USA, August 1977.

3. Sos, John Y., "Data Processing in the Instrument Telemetry Packet Era," Report X-560-77-192, Goddard Space Flight Center, Greenbelt, Maryland, USA, July 1977. 
4. Greenberg, Edward and Hooke, Adrian J., "Packet Telemetry: A Possible Standard Protocol for Spacecraft Data Handling," paper presented at Instrument Society of America Conference, Jet Propulsion Laboratory, Pasadena, California, USA, November 1978.

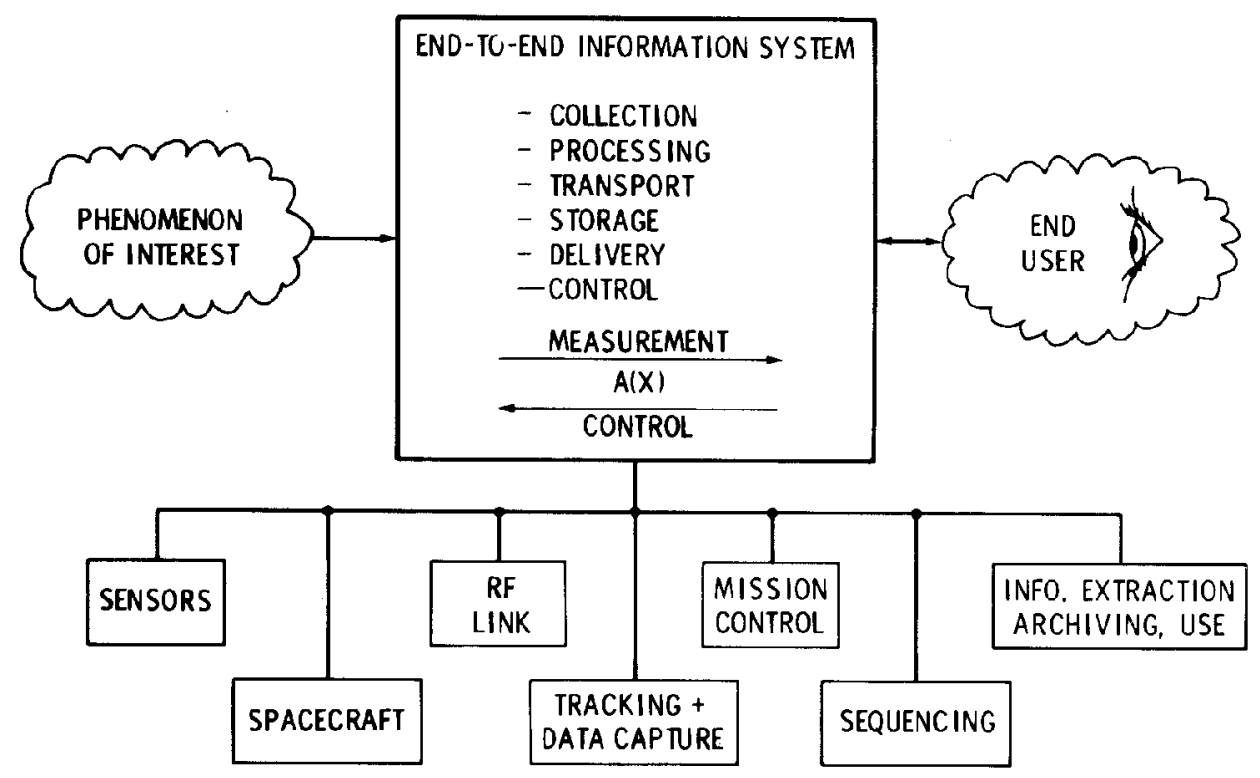

Figure 1 - Scope of the EEIS Transfer Function

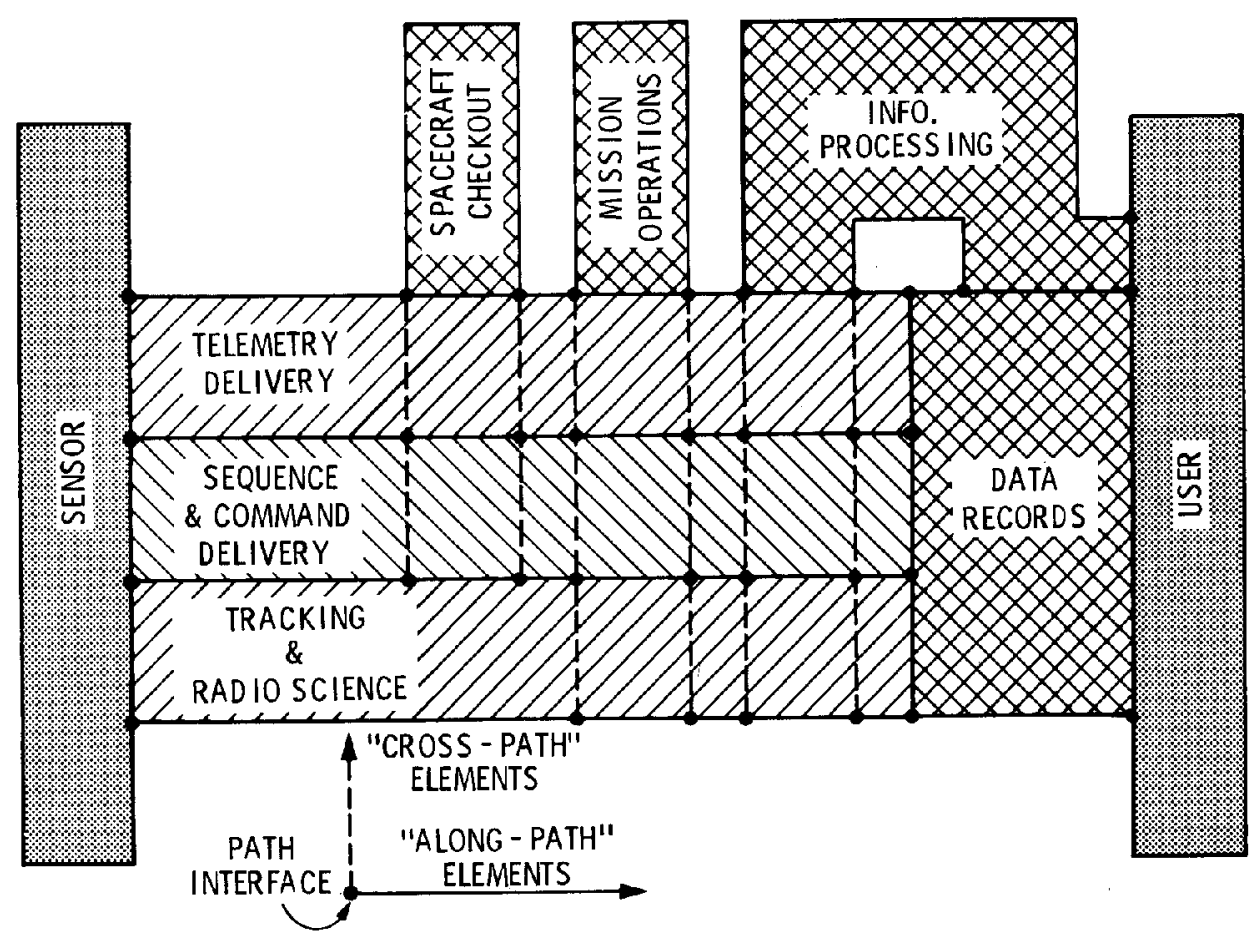

Figure 2 - Functional Elements of the JPL-EEIS 


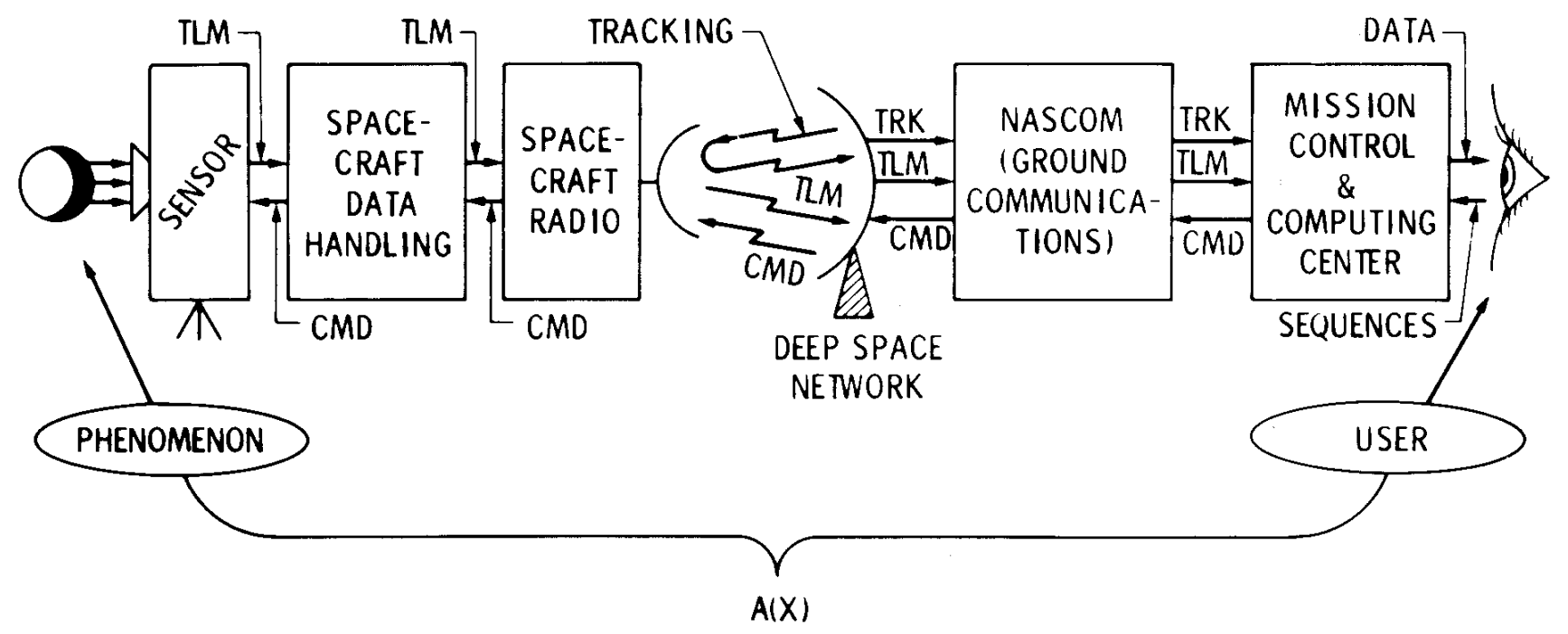

Figure 3 - Physical Elements of the EEIS 